\title{
Second trimester uterine rupture following medical termination of pregnancy: role of radiology in early diagnosis and prompt management
}

\begin{abstract}
A 27-year-old female patient presented with features of septicemic shock. She had recent history of medical abortion in second trimester followed by curettage. Ultrasound pelvis revealed heterogeneously echogenic contents in the endometrial cavity, which appears to be communicating with the collection in right adnexa, suggestive of uterine rupture with air containing abscess. It was confirmed on CT scan, which showed large right lateral uterine wall defect with communicating endometrial and pelvic air containing abscesses along with a heterogeneous mass containing macerated fetal parts resembling fetal skull in right adnexa.
\end{abstract}

Keywords: Uterine rupture, Medical termination of pregnancy, Radiology, Ultrasound, CT Scan

\author{
Volume 7 Issue 3 - 2017 \\ Jitendra Parmar, ${ }^{1}$ Maulik Vora, ${ }^{2}$ Chander \\ Mohan, ${ }^{3}$ Vikash Kumar' \\ Radiology Department, Dr. B. L. Kapur Memorial Hospital, India \\ 2Radiology Department, Indira Gandhi Medical College, India \\ ${ }^{3}$ Interventional Radiology Department, Dr. B. L. Kapur Memoria \\ Hospital, India
}

Correspondence: Jitendra Parmar, Radiology Department, Dr. B. L. Kapur Memorial Hospital, New Delhi, India, Tel 91 9643158267,Email jitendra32@gmail.com

Received: May 02, 2017 | Published: June 01, 2017
Abbreviations: CT scan, Computed Tomography; mm hg, millimeter of mercury; min, minute; ${ }^{\circ} \mathrm{C}$, degree Celsius; PCV, Packed Cell Volume

\section{Introduction}

Uterine rupture is extremely rare during early pregnancy. Patient usually presents with features of abdominal or pelvic infection or in more severe cases sepsis. Early diagnosis and prompt surgical management is of utmost importance. Ultrasound and cross sectional imaging can be of very helpful in diagnosing the condition and associated abnormalities.

\section{Case report}

A 27-year married female presented with complaints of high-grade fever and lower abdominal pain. She had history of spontaneous abortion at her home during 16th week of her pregnancy. Following which she underwent an elective curettage procedure in a private clinic. At the time of presentation, she was in hypotensive shock presumably due to ongoing septicemia. Her vitals were; pulse rate of $110 / \mathrm{min}$, blood pressure of $90 / 60 \mathrm{~mm} \mathrm{Hg}$ and respiratory rate of $32 / \mathrm{min}$. She had high-grade fever with body temperature of $39.2^{\circ} \mathrm{C}$. Abdominal examination showed generalized tenderness, guarding and rigidity. On pelvic examination, the uterus was enlarged, of approximately 12 weeks size and slightly tender. Per speculum examination showed closed internal os with non-smelly mild discharge was present. Her hemoglobin level was $5 \mathrm{~g} / \mathrm{dl}$, for which she received 4 units of PCV at private clinic. Clinical suspicion of endometritis or retained products of conception was made and ultrasound of pelvic organs was requested.

Trans-abdominal ultrasound of pelvic organs (Figure 1) revealed bulky uterus with defect in right postero-lateral wall (3 $\mathrm{cm}$ in maximum transverse diameter) of uterus, with an irregular heterogenous collection in right adnexa, which was communicating with endometrial cavity. Multiple tiny internal echoes were present in the collection, likely due to air, suggestive of infected collection. For confirmation and proper characterization non-contrast CT scan was performed.

Non-contrast CT scan of pelvis (Figure 2A) confirmed the defect in right lateral wall of uterus (thick red arrow) with multiple loculated air containing collections in right adnexa (curved blue arrow) and endometrial cavity (thin yellow arrow). Right adnexa showed heterogeneous solid appearing mass with presumably fetal parts including head (red circle in Figure 2B).

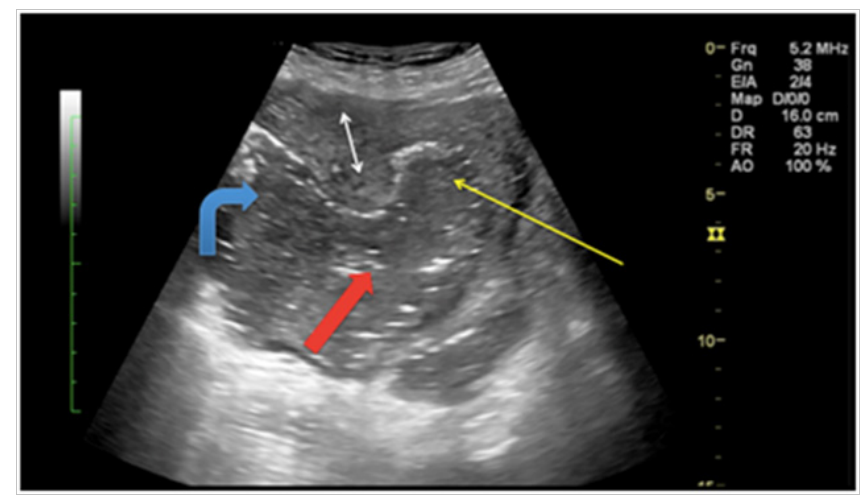

Figure I Trans-abdominal ultrasound shows defect in the right posterolateral wall of uterus (thick red arrow) with echogenic fluid filled collection in endometrial cavity (thin yellow arrow), which is communicating with collection in right adnexa (curved blue arrow). Right double arrow represents normal myometrium.

On laparotomy dirty foul smelling fluid was found in abdominal cavity. A pelvic abscess was seen on right side of pelvic cavity with few small bowel adhesions. Drainage of pelvic abscess revealed macerated incomplete fetal parts including deformed head.

\section{Discussion}

Uterine perforation is uncommon but potentially life threatening medical condition. It more commonly occurs during vaginal delivery especially in patients with previous cesarean delivery. Rupture of 
unscarred uterus in early pregnancy is extremely rare condition. Some other risk factors include external pressure, uterine contraction augmentation drugs, traumatic uterine conditions i.e. cesarean section, curettage procedure, abnormal placentation (accreta, increta and percreta), laparoscopic myomectomy. ${ }^{1,2}$

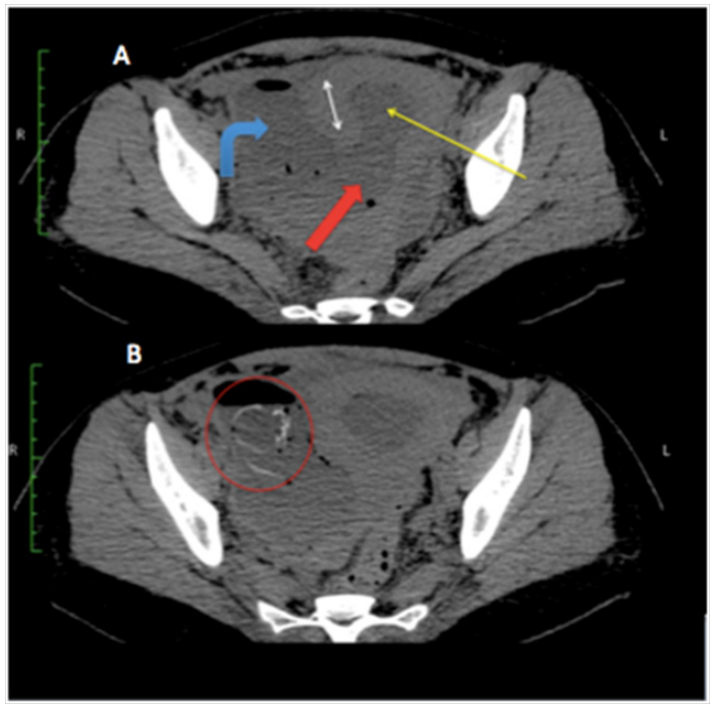

Figure 2 Non-contrast CT scan of pelvis; Figure 2A revealed the defect in right lateral wall of uterus (thick red arrow) with multiple loculated air containing collections in right adnexa (curved blue arrow) and endometrial cavity (thin yellow arrow). Right adnexa showed heterogeneous fluid and air filled collection with presumably fetal parts including head (red circle in Figure 2B).

Relatively avascular midline areas are more susceptible for perforation, however iatrogenic perforation like trauma from curettage can occur anywhere. ${ }^{3}$ Larger laterally situated defect carries poorer prognosis.

Ultrasound features of rupture are direct visualization of discontinuity in uterine wall. Other features include extra uterine fetal or placental parts, pelvic or abdominal collections, pneumoperitoneum, air or collection in endometrium, loops of bowel or large hematoma in myometrium. ${ }^{4,5}$ However, at times due to fetal or placental products, air containing abscesses or adynamic bowel containing gases Ultrasound do not provide optimal evaluation.
Cross sectional imaging techniques like CT scan can be used for better characterization and optimal surgical planning. CT scan may demonstrates heterogeneous fluid collections with or without air specks located in pelvic cavity or abdomen. ${ }^{3}$

\section{Conclusion}

Uterine rupture is a life threatening condition and it is uncommon in early pregnancy. This case highlights residual extra uterine extruded fetal parts with abdominal and pelvic abscesses. Cross sectional imaging like $\mathrm{CT}$ and MRI can help in diagnosis of this condition apart from Ultrasound.

\section{Acknowledgments}

None.

\section{Conflicts of interest}

None.

\section{References}

1. Sun HD, Su WH, Chang WH, et al. Rupture of a pregnant unscarred uterus in an early secondary trimester: A case report and brief review. $J$ Obstet Gynaecol Res. 2012;38(2):442-445.

2. Parker WH, Einarsson J, Istre O, et al. Risk Factors for Uterine Rupture after Laparoscopic Myomectomy. J Minim Invasive Gynecol. 2010;17(5):551-554.

3. Themistoklis SN, Chrysovalantis V, Stylianos A, et al. CT Diagnosis of an Abortion-Related Retroperitoneal Space Abscess. J Clin Med Res. 2011;3(5):268-269.

4. Sherer DM, Gorelick C, Gabbur N, et al. Transvaginal sonographic findings of a large intramural uterine hematoma associated with iatrogenic injury sustained at termination of pregnancy. Ultrasound Obstet Gynecol. 2007;30(1):110-113.

5. Crosfill FM, Hughes S. Ultrasound scan appearance of perforated uterus after surgical evacuation of retained products of conception. J Obstet Gynaecol. 2006;26(3):278-279. 